\title{
Impact of Vascular Anomalies on Pancreatoduodenectomy Procedure
}

\author{
Subhi Mansour ${ }^{\mathrm{a}}$, Mira Damouny ${ }^{\mathrm{a}}$, Miriam Obeid ${ }^{\mathrm{a}}$, Amir Farah ${ }^{\mathrm{a}}$, Kenan Halloun ${ }^{\mathrm{a}}$, \\ Rozan Marjiyeh ${ }^{a}$, Jawad Ghalia ${ }^{a}$, Yoram Kluger ${ }^{a}$, b, Safi Khuria, b, c
}

\begin{abstract}
Background: One of the critical steps during pancreatoduodenectomy (PD) procedure lies in identifying the complicated vascular anatomy of the resected area. The blood supply usually stems from branches of the celiac and the superior mesenteric arteries. However, only in $55-79 \%$ of surgeries, the anatomy of the blood vessels encountered by the surgeon is considered normal, while in the remaining cases, there are vascular variations that make these surgeries even more challenging. Any change or deviation from the known surgical course of PD makes surgery difficult and can result in an increase in intra/postoperative complications. In order to reduce difficulties encountered during PD, as well as reducing complication rates and improving surgical outcomes, a preliminary design, which includes preoperative identification of anatomical variations, is needed. The most accurate and accessible tool for identifying such variations is computed tomographic angiography (CTA). The aim of this retrospective study is to assess the prevalence of vascular anomalies encountered during PD, and examine whether there is an association between these anomalies and intra/postoperative morbidity and mortality.
\end{abstract}

Methods: A retrospective study over 5 years was performed at the HPB and Surgical Oncology Unit, Rambam Health Care Campus, Haifa, Israel. The charts of all patients submitted to PD were reviewed, and all patients with vascular anomalies were included. The types of anatomical variant as well as other information were collected. For statistical purposes, the group of patients with vascular anomalies was compared to a control group with identical demographic characteristics. A statistical analysis on possible association between vascular anomalies and intra/postoperative complications (mainly bleeding) was performed.

Results: During the aforementioned period, 202 patients underwent PD, and in $41(20.3 \%)$ vascular anomalies were identified. The ma-

Manuscript submitted February 18, 2021, accepted February 25, 2021

Published online March 19, 2021

${ }^{a}$ General Surgery Department, Rambam Health Care Campus, Haifa, Israel bHPB and Surgical Oncology Unit, Rambam Health Care Campus, Haifa, Israel

${ }^{c}$ Corresponding Author: Safi Khuri, HPB and Surgical Oncology Unit, General Surgery Department, Rambam Health Care Campus, Haa'leya Hashniya, Haifa 31096, Israel. Email: s_khuri@rambam.health.gov.il

doi: https://doi.org/10.14740/jocmr4455 jority of these patients (32/41) had single anatomical variant, where two and three anatomical variants were identified in seven and two patients, respectively. The most common vascular anomaly was replaced right hepatic artery, noticed in 34 patients $(83 \%)$. The most common indication for PD for both groups (anatomical variant and control group) was pancreas adenocarcinoma (56\%). Intraoperative complication, in the form of bleeding, developed in $6 / 41$ patients $(14.6 \%)$ of the anatomical variant group and none in the control group. Postoperative complication rates, including post- pancreatoduodenectomy hemorrhage ( $\mathrm{PPH})$, postoperative pancreatic fistula (POPF), intra-abdominal abscess and wound infection were almost identical for both groups. One case of death within 30 days occurred, and it was in the anatomical variant group.

Conclusions: Anatomical vascular anomalies are a common variant encountered during PD, with RRHA being the most common. Although postoperative morbidity and mortality are not affected by the presence of these anomalies, intraoperative bleeding rate is higher in this specific group, thus; a preoperative diagnosis by means of CTA is mandatory.

Keywords: Pancreatoduodenectomy; Vascular anomalies; Post-pancreatoduodenectomy complications; Post-pancreatoduodenectomy hemorrhage

\section{Introduction}

Pancreatoduodenectomy (PD), firstly reported by Allen Whipple in 1945 [1], is the standard surgical procedure for the management of benign and malignant diseases of the peri-ampullary region (head of the pancreas, ampulla of Vater, distal common bile duct and second part of the duodenum), highly selective cases of chronic pancreatitis and severe trauma to the pancreatic head and duodenum $[2,3]$. It is complex and highly challenging operation for surgeons. Although mortality rates following PD have fallen to less than $1 \%$ due to the development of specialized centers, along with the improvement in perioperative care and the advanced progression of the used surgical techniques [4-6], morbidity rates remains high at about $30-40 \%$ [7, 8]. Complications following PD are diverse and include postoperative pancreatic fistula (POPF), delayed gastric emptying (DGE), intra-abdominal collections, surgical site infection (SSI) and post-PD hemorrhage (PPH), with the 
latter being the least frequent complication following PD. One of the critical steps during PD procedure lies in identifying the complicated vascular anatomy of the resected area, which is usually branch of the celiac and superior mesenteric arteries. It is well known that the normal vascular anatomy for the resected area is encountered in only $55-79 \%$ of patients, while in the remaining cases there are vascular, especially arterial, variations that add to the surgical challenge during PD [9-11]. The presence of said anatomical variations may increase the risk of complications through direct (bleeding due to iatrogenic intraoperative vessel injury) or indirect (postoperative ischemia of tissues and anastomotic leakage) mechanisms. The course of these abnormal vascular anomalies varied according to their origin; Replaced/accessory right hepatic arteries arising from the superior mesenteric artery (SMA) usually pass posterolateral to the portal vein and enter the hepatoduodenal ligament posterolateral to the common bile duct. Some vascular anomalies (e.g., replaced right hepatic artery (RRHA)) may alter the operation course, while others (e.g., replaced left hepatic artery (RLHA), accessory hepatic artery (AHA)) usually do not. According to the Hiatt classification of the surgical anatomy of the hepatic artery, the most common anomalies include a replaced or accessory right hepatic artery without (type III, $10.6 \%$ ) or with (type IV, 2.3\%) an accessory or RLHA [12]. As the failure to preoperative recognition of such anomalies may increase the risk for accidental iatrogenic vascular injury, the understanding of such vascular anomalies by means of preoperative contrast-enhanced computed tomography (CT) scan is of great interest to guide the surgeon during PD procedure in order to minimize the risk for intra/postoperative complications, especially hemorrhage.

The aim of this retrospective study is therefore to assess the prevalence of vascular anomalies encountered during PD and examine whether these anomalies affect intra/postoperative morbidity and mortality rates.

\section{Materials and Methods}

A retrospective study over 5 years, between January 1, 2014 and December 31, 2018 was performed at the Hepato-Pancreato-Biliary (HPB) Unit of the General Surgery Department at Rambam Health Care Campus, Haifa, Israel. All patients aged 18 years and over, who underwent PD, were included in the study.

Patients who underwent PD due to severe trauma to the pancreatic head or duodenum, as well as other form of pancreatic resection, such as distal or total pancreatectomy, were excluded from the study. Patients were checked for re-admission to our hospital or to other medical centers.

For statistical analysis, the group of patients with anatomical vascular anomalies was compared to a control group with identical demographic and surgical characteristics.

The main outcome was association between vascular anomalies and intra/postoperative hemorrhage. Other collected information including number and types of vascular anomalies, indication for operation, possible association for other surgical complications (e.g., POPF, intra-abdominal abscess, delayed gastric emptying) and mortality was also registered.

\section{Ethical issues and informed consent}

This article does not contain any experimental studies with human participants or animals performed by any of the authors. The study was approved by the Ethical Committee. Due to the retrospective nature of the study, informed consent was waived. The study was approved by the Review Board of our Department.

\section{Statistics}

Continuous variables were summarized as mean and standard deviation (SD), discrete variables were categorically expressed by numbers and percentages. A Student's $t$-test was used for continuous variables. A Fisher's exact test, depending on the results, was used for variables that were dichotomous. A $P$ value of less than 0.05 was considered significant.

Worth mentioning, none of the included patients was evaluated for anatomical vascular anomaly by means of abdominopelvic contrast-enhanced CT scan preoperatively. Patients were diagnosed with vascular anatomical variant during operations.

\section{Surgical approach}

The two main approaches for PD procedure are the "conventional" approach and the "artery first" one. The typical conventional approach usually begins with Kocher's maneuver, to exfoliate the pancreatic head and duodenum from the retroperitoneum, followed by lymphadenectomy, division of the stomach, bile duct and pancreas, and finally dissection of the connective tissue around the SMA. The concept of the recently described "artery first" approach is to start the dissection of the connective tissues around the SMA, which may decrease intraoperative blood loss by early control of blood flow to the pancreatic head by division of the inferior pancreatoduodenal artery in an early stage.

At our institution, we usually use the typical conventional approach for PD procedures.

\section{Results}

During the study period, 202 patients underwent PD for the management of periampullary diseases. Forty-one patients $(20.3 \%)$ were identified with anatomical vascular anomalies. For statistical purposes, the group of patients with anatomical variants was compared to a control group of patients with similar demographic and surgical characteristics (Table 1). The majority of the anatomical variant group patients (32/41) had single anatomical variant, where two and three anatomical variants were identified in seven and two patients, respectively. The most common vascular anomaly was RRHA noticed in 
Table 1. Summary of the Demographic and Surgical Characteristics of the Variant as Well as the Control Group of Patients

\begin{tabular}{|c|c|c|}
\hline & Anatomical variant group & Control group \\
\hline Number of patients & 41 & 41 \\
\hline \multicolumn{3}{|l|}{ Gender } \\
\hline Female & 21 & 19 \\
\hline Average age (years) & 64 & 65 \\
\hline PDAC & 23 & 22 \\
\hline Ampullary adenocarcinoma & 8 & 7 \\
\hline $\mathrm{CC}$ & 2 & 1 \\
\hline IgG4 pancreatitis & 2 & 0 \\
\hline Duodenal villous adenoma & 2 & 2 \\
\hline IPMN & 2 & 4 \\
\hline Duodenal GIST & 0 & 1 \\
\hline Anticoagulant therapy & 3 & 1 \\
\hline Antiplatelets therapy & 13 & 13 \\
\hline Previous abdominal surgery & 5 & 6 \\
\hline Average BMI & 27.4 & 28.2 \\
\hline
\end{tabular}

PDAC: pancreatic ductal adenocarcinoma; CC: cholangiocarcinoma; IgG4: immunoglobulin G4; SPPT: solid pseudopapillary tumor; NET: neuroendocrine tumor; IPMN: intraductal papillary mucinous neoplasm; GIST: gastrointestinal stromal tumor; BMI: body mass index.

34 patients $(83 \%)$, and originated from the SMA, gastroduodenal artery (GDA) and celiac trunk in 29, four and one patients, respectively. The second most common anatomical vascular variant was RLHA found in eight patients (19.5\%), originating from left gastric artery (LGA), followed by replaced gastroduodenal artery (RGDA) and AHA noticed in three patients each (Table 2). Of patients with multiple variants, the most common vascular anomalies were RRHA (originating from SMA) and RLHA (originating from LGA) described in five patients. The most common indication for PD was pancreatic ductal adenocarcinoma (PDAC, 56\%), followed by ampullary adenocarcinoma (19\%). About $39 \%$ of patients were regularly treated by antiplatelets/anticoagulant therapy, and only $12 \%$ have a history of previous abdominal surgeries.

As reported in Table 3, for statistical analysis, patients were grouped into anatomical variant and control groups. Intraoperative hemorrhage developed in six patients $(15 \%)$ of the anatomical variant group and in none of the control group. This was statistically significant when using Fisher's exact test, with $\mathrm{P}$ value of 0.026 , indicating an association between vascular anomalies and intraoperative bleeding. On the other hand, no significant association was found between the differ-

Table 2. Patients With Anatomical Vascular Variants

\begin{tabular}{|c|c|c|}
\hline Anatomical vascular variant & Origin & Number of patients \\
\hline \multirow[t]{3}{*}{ Replaced right hepatic artery } & Superior mesenteric artery & 29 \\
\hline & Gastroduodenal artery & 4 \\
\hline & Celiac artery & 1 \\
\hline Replaced left hepatic artery & Left gastric artery & 8 \\
\hline \multirow[t]{2}{*}{ Replaced gastroduodenal artery } & Celiac artery & 2 \\
\hline & Superior mesenteric artery & 1 \\
\hline Accessory hepatic artery & Superior mesenteric artery & 3 \\
\hline Replaced gastroepiploic artery & Celiac artery & 2 \\
\hline Replaced middle colic artery & Gastroduodenal artery & 2 \\
\hline
\end{tabular}


Table 3. Intra/Postoperative Complications

\begin{tabular}{|c|c|c|c|}
\hline & Anatomical variant group $(n=41)$ & Control group $(n=41)$ & P value \\
\hline Intraoperative bleeding & 6 & 0 & 0.026 \\
\hline Bilio-enteric leak & 0 & 0 & $\mathrm{~N} / \mathrm{A}$ \\
\hline Postoperative pancreatic fistula & 8 & 12 & 0.441 \\
\hline Delayed gastric emptying & 6 & 7 & 0.773 \\
\hline Re-operation in 30 days & 0 & 0 & N/A \\
\hline Mortality in 30 days & 1 & 0 & 0.5 \\
\hline
\end{tabular}

N/A: not applicable.

ent types of vascular anomalies encountered and postoperative complications, including PPH, POPF, DGE, SSI and intra-abdominal abscess. In our study, the overall rate of $\mathrm{PPH}$ was $9 \%$ in the anatomical variant group and $7 \%$ in the control group, with no statistically significant difference. All cases of PPH were late (more than $24 \mathrm{~h}$ after operation) and mild (smallmedium blood loss, decrease in hemoglobin levels of less than $3 \mathrm{~g} / \mathrm{dL}$ ), according to the International Study Group of Pancreatic Surgery (ISGPS). Although the difference of the rate of POPF in patients with and without vascular anomalies was $10 \%$, the association between POPF and vascular anomalies was not statistically significant with a $P$ value of 0.441 . The cases of POPF were registered according to the classification reported by Bassi et al [13], with grade A POPF being replaced by biochemical leak. All cases were of the biochemical leak.

There was no difference in rates of DGE, SSI and intraabdominal abscess formation between the two groups, indicating lack of association between vascular anomalies and the aforementioned complications. Moreover, no cases of hepatico-jejunal anastomotic leakage reported in both groups.

No cases of re-operation within 30 days were reported in both groups, and only one case of 30 days' mortality was reported in the anatomical variant group.

The estimated blood loss for the anatomical variant group was about $500 \mathrm{~mL}$, while it was $50 \mathrm{~mL}$ for the control group.

\section{Discussion}

The results of this study show that $20 \%$ of patients undergoing PD $(n=202)$ have anatomical vascular anomaly, and that was significantly associated with higher rates of intraoperative hemorrhage. This percentage is far less than the percentage reported in the English literature [9-11].

Numerous anatomical variants exist between the main three arteries supplying the gastrointestinal tract: celiac trunk, SMA and inferior mesenteric artery, most of which regress during embryological development. Thus, vascular anomalies may commonly be encountered during other abdominal surgeries, other than PD.

The study is retrospective, but all consecutive patients treated in the aforementioned years have been included, patient's data were stored in a database that allows easy selection of cases of interest based on the diagnosis, intervention and postoperative complications. Rambam Health Care Campus is a referral center for 10 other hospitals that give medical cover for 2 million citizens, especially for all patients of which major pancreatic surgery is indicated. The study should therefore be considered as having a rather low risk of selection bias.

There were no racial differences in the vascular as well as the control groups, as most patients included were Caucasian.

RRHA arising from the SMA is the most common anatomical vascular variant in the hepatic arterial anatomy, reported to occur in $11-22 \%$ of patients $[12,14,15]$. The same findings were demonstrated in this study, with RRHA arising from the SMA being the most common, found in $70 \%$ of patients with vascular anomalies. In his report, Balachandran et al demonstrated an increased risk for intraoperative vascular complications and poor outcome in patients with RRHA undergoing PD [15]. Due to the fact that RHA is the main blood supply to the bile ducts, accidental injury of such artery could lead to ischemia of the hepatico-enteric anastomosis and leakage during the postoperative period [16]. In our study, no cases of bilio-enteric anastomosis leakage were reported in both groups. Moreover, Asano et al evaluated the feasibility of concomitant resection of RRHA detected while performing PD without preoperative embolization or intraoperative reconstruction and showed no association with severe morbidity [17].

The second most common vascular anomaly in this study was RLHA (19.5\%). Although no direct postoperative complication was associated with this anomaly, Yamamoto et al describes two cases in which liver necrosis was shortly observed following PD with resection of a RLHA [18], one of which necessitated lateral segmentectomy of the liver.

Upon taking the full range of vascular anomalies encountered during PD in this study into consideration, the sole outcome with a statistical significance is intraoperative bleeding $(\mathrm{P}<0.05)$, indicating the importance of presurgical identification and preparation for the vascular anomalies. No other study contemplating vascular anomalies during PD found a statistically significant correlation with intraoperative bleeding. Hence, suggesting the need for further studies. Regarding 
hemorrhage observed postsurgically, as mentioned before, $9 \%$ of cases had PPH in the vascular anomaly group compared with $7 \%$ in the study group, with no statistical significance. A postoperative hemorrhage is an uncommon complication after PD but is one of the major causes of perioperative mortality [19]. Based on ISGPS, the incidence of postoperative hemorrhage is about 4-16\% [20]; other studies showed similar rates [21-25]. Hence, PPH incidence in our study falls within these limits, and it is safe to conclude that vascular anomalies had no impact on the development of this complication.

There were no other postsurgical complications associated with vascular anomalies, including POPF, DGE, SSI and intra-abdominal abscess formation. Several studies reporting on vascular anomalies and PD failed to report on postoperative complications [26, 27]. Perwaiz et al analyzed the clinical impact of vascular anomalies on the procedure itself; the study included 200 patients, $26.5 \%$ of which had arterial anomalies. The study indicated that arterial anomalies could increase operative complexity but do not usually compromise the safety of the procedure or its oncological outcome [28].

The emphasis on presurgical identification of vascular anomalies cannot be overstated, allowing the surgeon to prepare beforehand for possible complications (such as intraoperative bleeding), one of the most used modalities for diagnosis and identifications of vascular anatomy is multi-detector CT (MDCT) angiography [29]. Subbiah Nagaraj et al demonstrated a $100 \%$ statistical correlation between imaging and surgical findings when assessing MDCT angiography for presurgical anatomical variations identification [30]. Another study by Yang et al demonstrated similar results with MDCT being less sensitive for detecting celiac artery stenosis [31]. Whereas Staskiewicz et al showed that detection and evaluation of vascular anomalies does not necessarily require angiography-CT, rather a MDCT is sufficient to provide the knowledge of the HA abnormalities [32].

\section{Conclusions}

Vascular anomalies of the celiac and SMA branches are relatively common, such anomalies can alter the course of the surgery causing an increase if intraoperative complication rate. Hence, the use of various detection radiographic modalities such as MDCT angiography for documenting such anomalies prior to the surgery is of clinical importance. Due to the small number of patients $(n=41)$ with vascular anomalies included in this study, a high volume multi-centric studies are encouraged.

\section{Acknowledgments}

None to declare.

\section{Financial Disclosure}

There was no specific funding source to be mentioned.

\section{Conflict of Interest}

The authors have no conflicts of interest to declare.

\section{Informed Consent}

Due to the retrospective nature of the study, informed consent was waived.

\section{Author Contributions}

Acquisition of the data was made by Mira Damouny, Subhi Mansour and Miriam Obaid; analysis and interpretation were done by Safi Khuri, Rouzan Marjeya and Kenan Halloun. The paper was drafted by Safi Khuri, Jawad Ghalia and Subhi Mansour; critical revision and final approval of the published version were done by Safi khuri and Yoram Kluger. All authors agree to be accountable for all aspects of the work in ensuring that questions related to the accuracy or integrity of any part of the work are appropriately investigated and resolved.

\section{Data Availability}

The authors declare that data supporting the findings of this study are available within the article.

\section{References}

1. Whipple AO. Pancreaticoduodenectomy for islet carcinoma: a five-year follow-up. Ann Surg. 1945;121(6):847852.

2. Scaife CL, Hewitt KC, Mone MC, Hansen HJ, Nelson ET, Mulvihill SJ. Comparison of intraoperative versus delayed enteral feeding tube placement in patients undergoing a Whipple procedure. HPB (Oxford). 2014;16(1):6269.

3. Leichtle SW, Kaoutzanis C, Mouawad NJ, Welch KB, Lampman R, Hoshal VL, Jr., Kreske E. Classic Whipple versus pylorus-preserving pancreaticoduodenectomy in the ACS NSQIP. J Surg Res. 2013;183(1):170-176.

4. Neoptolemos JP, Russell RC, Bramhall S, Theis B. Low mortality following resection for pancreatic and periampullary tumours in 1026 patients: UK survey of specialist pancreatic units. UK Pancreatic Cancer Group. Br J Surg. 1997;84(10):1370-1376.

5. Shrikhande SV, Barreto SG, Somashekar BA, Suradkar K, Shetty GS, Talole S, Sirohi B, et al. Evolution of pancreatoduodenectomy in a tertiary cancer center in India: improved results from service reconfiguration. Pancreatology. 2013;13(1):63-71.

6. Trede M, Schwall G, Saeger HD. Survival after pancreatoduodenectomy. 118 consecutive resections without an operative mortality. Ann Surg. 1990;211(4):447-458.

7. Bassi C, Falconi M, Salvia R, Mascetta G, Molinari E, 
Pederzoli P. Management of complications after pancreaticoduodenectomy in a high volume centre: results on 150 consecutive patients. Dig Surg. 2001;18(6):453-457; discussion 458.

8. Gouma DJ, van Geenen RC, van Gulik TM, de Haan RJ, de Wit LT, Busch OR, Obertop H. Rates of complications and death after pancreaticoduodenectomy: risk factors and the impact of hospital volume. Ann Surg. 2000;232(6):786-795.

9. Koops A, Wojciechowski B, Broering DC, Adam G, Krupski-Berdien G. Anatomic variations of the hepatic arteries in 604 selective celiac and superior mesenteric angiographies. Surg Radiol Anat. 2004;26(3):239-244.

10. Covey AM, Brody LA, Maluccio MA, Getrajdman GI, Brown KT. Variant hepatic arterial anatomy revisited: digital subtraction angiography performed in 600 patients. Radiology. 2002;224(2):542-547.

11. Michels NA. Newer anatomy of the liver and its variant blood supply and collateral circulation. Am J Surg. 1966;112(3):337-347.

12. Hiatt JR, Gabbay J, Busuttil RW. Surgical anatomy of the hepatic arteries in 1000 cases. Ann Surg. 1994;220(1):5052.

13. Bassi C, Marchegiani G, Dervenis C, Sarr M, Abu Hilal M, Adham M, Allen P, et al. The 2016 update of the International Study Group (ISGPS) definition and grading of postoperative pancreatic fistula: 11 Years After. Surgery. 2017;161(3):584-591.

14. Rong GH, Sindelar WF. Aberrant peripancreatic arterial anatomy. Considerations in performing pancreatectomy for malignant neoplasms. Am Surg. 1987;53(12):726729.

15. Balachandran A, Darden DL, Tamm EP, Faria SC, Evans $\mathrm{DB}$, Charnsangavej C. Arterial variants in pancreatic adenocarcinoma. Abdom Imaging. 2008;33(2):214-221.

16. Biehl TR, Traverso LW, Hauptmann E, Ryan JA, Jr. Preoperative visceral angiography alters intraoperative strategy during the Whipple procedure. Am J Surg. 1993;165(5):607-612.

17. Asano T, Nakamura T, Noji T, Okamura K, Tsuchikawa T, Nakanishi Y, Tanaka K, et al. Outcome of concomitant resection of the replaced right hepatic artery in pancreaticoduodenectomy without reconstruction. Langenbecks Arch Surg. 2018;403(2):195-202.

18. Yamamoto $M$, Zaima $M$, Yamamoto $H$, Harada $H$, Kawamura J, Yamada M, Yazawa T, et al. Liver necrosis shortly after pancreaticoduodenectomy with resection of the replaced left hepatic artery. World J Surg Oncol. 2017;15(1):77.

19. Zhou TY, Sun JH, Zhang YL, Zhou GH, Nie CH, Zhu TY, Chen SQ, et al. Post-pancreaticoduodenectomy hemorrhage: DSA diagnosis and endovascular treatment. Oncotarget. 2017;8(43):73684-73692.

20. Welsch T, Eisele H, Zschabitz S, Hinz U, Buchler MW, Wente MN. Critical appraisal of the International Study
Group of Pancreatic Surgery (ISGPS) consensus definition of postoperative hemorrhage after pancreatoduodenectomy. Langenbecks Arch Surg. 2011;396(6):783791.

21. Feng J, Chen YL, Dong JH, Chen MY, Cai SW, Huang ZQ. Post-pancreaticoduodenectomy hemorrhage: risk factors, managements and outcomes. Hepatobiliary Pancreat Dis Int. 2014;13(5):513-522.

22. Zhang L, Wang J, Jiang J, Shen J. The Role of Interventional Radiology in the Management of Late Postpancreaticoduodenectomy Hemorrhage. Biomed Res Int. 2020;2020:8851950.

23. Manas-Gomez MJ, Rodriguez-Revuelto R, Balsells-Valls J, Olsina-Kissler JJ, Caralt-Barba M, Perez-Lafuente M, Charco-Torra R. Post-pancreaticoduodenectomy hemorrhage. Incidence, diagnosis, and treatment. World J Surg. 2011;35(11):2543-2548.

24. Wellner UF, Kulemann B, Lapshyn H, Hoeppner J, Sick O, Makowiec F, Bausch D, et al. Postpancreatectomy hemorrhage - incidence, treatment, and risk factors in over 1,000 pancreatic resections. J Gastrointest Surg. 2014;18(3):464-475.

25. Tien YW, Lee PH, Yang CY, Ho MC, Chiu YF. Risk factors of massive bleeding related to pancreatic leak after pancreaticoduodenectomy. J Am Coll Surg. 2005;201(4):554-559.

26. Yang F, Long J, Fu DL, Jin C, Yu XJ, Xu J, Ni QX. Aberrant hepatic artery in patients undergoing pancreaticoduodenectomy. Pancreatology. 2008;8(1):50-54.

27. Ye Z, Ye S, Zhou D, Zheng S, Wang W. A rare variation of celiac trunk and hepatic artery complicating pancreaticoduodenectomy: A case report and literature review. Medicine (Baltimore). 2017;96(48):e8969.

28. Perwaiz A, Singh A, Singh T, Chaudhary A. Incidence and management of arterial anomalies in patients undergoing pancreaticoduodenectomy. JOP. 2010;11(1):25-30.

29. Costea AN, Iacob N, Pusztai AM, Ples H, Matusz P. Replaced right hepatic artery arising from inferior pancreaticoduodenal artery, in association with left multiple renal arteries: a case report using MDCT angiography. Rom J Morphol Embryol. 2019;60(3):971-977.

30. Subbiah Nagaraj S, Kaman L, Dahiya D, Ramavath K, Kalra N, Behera A. Correlation of multi-detector computed tomography and intraoperative variations of the celiac trunk and hepatic artery in resectable hepatobiliary pancreatic cancers. Cureus. 2020;12(12):e12106.

31. Yang F, Di Y, Li J, Wang XY, Yao L, Hao SJ, Jiang YJ, et al. Accuracy of routine multidetector computed tomography to identify arterial variants in patients scheduled for pancreaticoduodenectomy. World J Gastroenterol. 2015;21(3):969-976.

32. Staskiewicz G, Torres K, Denisow M, Torres A, Czekajska-Chehab E, Drop A. Clinically relevant anatomical parameters of the replaced right hepatic artery (RRHA). Surg Radiol Anat. 2015;37(10):1225-1231. 